\title{
Blast from the Past-using PTH to Differentiate Acute versus Chronic Kidney Disease
}

\author{
Chase Cavayero', Sunny M Kar ${ }^{2}$ and Pran M Kar ${ }^{3 *}$ \\ ${ }^{1}$ Lake Erie College of Osteopathic Medicine, Erie, Pennsylvania, USA \\ ${ }^{2}$ Rowan University, New Jersey, USA \\ ${ }^{3}$ Veterans Adminstration, Orlando, Florida, USA
}

\begin{abstract}
In these times of limited financial and human resources, physicians must be cognizant of the rising costs of healthcare. Expensive imaging studies should be reserved for conditions that are particularly challenging to diagnose using traditional laboratory tests. Consequently, we must revisit the use of alternative simple, inexpensive and readily available laboratory tests to help differentiate medical conditions. Therefore, we would like to revisit the use of intact PTH as a discriminatory lab value in evaluating chronic vs. acute kidney injury.
\end{abstract}

Keywords: Intact PTH; Acute versus Chronic Kidney Failure; Hyperkalemia; Phosphorus

\section{Introduction}

In the absence of imaging studies, it is difficult to determine if elevated creatinine is the result of an acute or chronic kidney insult. Many lab values have been utilized to determine this complex question with varying success. Previously, it has been ascertained that hyperkalemia is one of the best indicators of CKD, as the kidney is able to adjust for decreased GFR (glomerular filtration rate) by increasing colonic excretion of potassium [1-4]. Carbamylated Hemoglobin has also been used to differentiate the two conditions, $[5,6]$ however this method has fallen out of favor due to the shortened red blood cell life span associated with uremia. Interestingly, intact parathyroid hormone (i-PTH) can be utilized similarly. However, the complex regulation of $\mathrm{PTH}$ in the setting of acute and chronic kidney injury has prevented its widespread use.

Assays that measure the full-length hormone comprised of 84 amino acids, or PTH (1-84), are known as "intact" PTH (i-PTH) assays [7]. Earlier radioimmunoassays commonly detected various fragments of PTH that accumulate in the serum/plasma of patients with CKD because their renal clearance and/or degradation are diminished. In contrast to the various fragments that are detected in more traditional assays, intact PTH is not metabolized by the kidney and should not give artificially high values [7].

The regulation of parathyroid hormone is a complex process involving the synchronization of the skeletal and endocrine systems as well as the kidneys. Not surprisingly, many peripheral disorders can affect PTH homeostasis resulting in secondary hyperparathyroidism (SHPT). Of these disorders, chronic kidney disease (CKD) is most commonly encountered [8]. Although CKD has long been associated with SHPT, the proposed mechanism for parathyroid dysfunction has been the subject of much debate. Nonetheless, several factors have been implicated in the pathogenesis.

CKD results in a dysregulation of calcium, phosphate, and vitamin D homeostasis. In this pathway, elevated phosphate concentration, low serum calcium concentration, and reduced $1,25(\mathrm{OH}) 2 \mathrm{D} 3$ (calcitriol) lead to increased PTH synthesis $[9,10]$. Further, a more recent development in the understanding of SHPT describes the role of phosphatonin fibroblast growth factor (FGF-23) in controlling PTH levels. In response to elevated phosphate, FGF-23 diminishes renal 25 $(\mathrm{OH})$-1-hydroxylase, a vital enzyme responsible for the conversion to active vitamin D [10,11]. Interestingly, FGF-23 dysregulation does not appear to be limited to CKD and has been observed in AKI $[12,13]$.
Further compounding the issue is a possible decrease in the activation of the vitamin D receptor [14].

Whatever the mechanism of altered homeostasis, elevated PTH has been linked to a variety of poor clinical outcomes reaching far beyond normal bone metabolism. These manifestations include increased risk for cardiovascular disease, decreased immune function, and an overall decreased quality of life $[15,16]$. Additionally, excess PTH appears to have neurotoxic effects as EEG abnormalities have been observed in patients with kidney failure [17]. Such toxic effects of PTH are proposed to occur secondary to an elevation of IL-6, C-reactive protein, and tumor necrosis factor- $\alpha$, thus creating a state of inflammation [18]

\section{Case 1}

A 35 year-old African American female with a past history of pituitary adenoma status post surgery and no history of renal disease presented with a 3 day history of excessive drowsiness. In the ER the patient was found to be severely hypotensive and in septic shock. Acute kidney injury likely due to volume depletion was considered after sudden onset of elevated creatinine. Additionally, despite normal serum calcium and phosphorous, labs revealed a PTH (intact) of $163 \mathrm{pg} / \mathrm{ml}$. The patient was continued on antibiotics and began to recover slowly (Table 1).

\section{Case 2}

A 69 year-old female with a history of hypertension and CVA was brought to the emergency department after falling and injuring her ankle. The patient was noted to have high creatinine and no history of renal disease prompting, renal consult. Acute kidney injury, likely due to volume depletion was considered. After a further rise in creatinine, i-PTH was noted to be elevated at $104 \mathrm{pg} / \mathrm{ml}$. The patient was started on IV fluids and monitored. The creatinine began to decrease and the patient recovered (Table 2).

*Corresponding author: Anitha Pran M Kar, Staff Nephrologist, Veterans Adminstration, 5201 Raymond, Street, Room \#1467, Orlando, Florida 32803 , USA, Tel: 407312 1533; Fax: 4078723990; E-mail: prankar@gmail.com

Received November 18, 2014; Accepted January 03, 2015; Published January 08, 2015

Citation: Cavayero C, Kar SM, Kar PM (2014) Blast from the Past-using PTH to Differentiate Acute versus Chronic Kidney Disease. J Nephrol Ther 5: 191. doi:10.4172/2161-0959.1000191

Copyright: (c) 2015 Cavayero C, et al. This is an open-access article distributed under the terms of the Creative Commons Attribution License, which permits unrestricted use, distribution, and reproduction in any medium, provided the original author and source are credited. 


\begin{tabular}{|c|c|c|c|c|c|}
\hline Case 1 & Day 1 & Day 2 & Day 2 & Day 3 & Day 5 \\
\hline Creatinine (mg/dL) & 3.85 & 3.34 & & 3.32 & 2.89 \\
\hline PTH(intact) pg/dL & & & 163 & & \\
\hline Calcium (mg/dL) & 10.1 & 8.5 & & 8.5 & 8.3 \\
\hline Phosphorus (mg/dL) & & & 2.5 & & \\
\hline Potassium (mg/dL) & 3.6 & 4.6 & & 3.9 & 3.5 \\
\hline
\end{tabular}

Table 1: A 35 year-old African American female with a past history of pituitary adenoma status post surgery.

\begin{tabular}{|c|c|c|c|c|c|}
\hline Case 2 & $\begin{array}{c}\text { Day 1 } \\
\mathbf{1 7 : 2 8}\end{array}$ & $\begin{array}{c}\text { Day 2 } \\
\mathbf{5 : 4 4}\end{array}$ & $\begin{array}{c}\text { Day 3 } \\
\mathbf{1 0 : 2 7}\end{array}$ & Day 4 & Day 5 \\
\hline Creatinine (mg/dL) & 3.42 & 3.43 & 2.25 & 1.82 & 1.49 \\
\hline PTH(intact) $\mathbf{~ g g / d L ~}$ & & 104 & & & \\
\hline Calcium (mg/dL) & 9.4 & 9.1 & 8.2 & 8.7 & 8.8 \\
\hline Phosphorus (mg/dL) & & 4.7 & & 3 & \\
\hline Potassium (mg/dL) & 5 & 5.3 & 4.5 & 5.5 & 4.6 \\
\hline
\end{tabular}

Table 2: A 69 year-old female with a history of hypertension and CVA.

\begin{tabular}{|c|c|c|c|c|c|}
\hline Case 3 & $\begin{array}{c}\text { Day 1 } \\
\mathbf{4 : 0 3}\end{array}$ & $\begin{array}{c}\text { Day 1 } \\
\mathbf{1 3 : 2 5}\end{array}$ & $\begin{array}{c}\text { Day 2 } \\
\mathbf{5 : 0 5}\end{array}$ & $\begin{array}{c}\text { Day 3 } \\
\mathbf{2 : 1 1}\end{array}$ & $\begin{array}{c}\text { Day 4 } \\
\mathbf{4 : 0 0}\end{array}$ \\
\hline Creatinine (mg/dL) & 4.19 & 3.36 & 2.72 & 1.89 & 1.60 \\
\hline PTH (intact) $\mathbf{~ g g / d L ~}$ & & & 75 & & \\
\hline Calcium (mg/dL) & 7.5 & 8.9 & 7.6 & 7.6 & 8.0 \\
\hline Phosphorus (mg/dL) & & & 2.7 & 2.2 & 2.6 \\
\hline Potassium (mg/dL) & 3.8 & 3.4 & 3.1 & & 2.9 \\
\hline
\end{tabular}

Table 3: A 55 year-old female with no history of renal disease.

\begin{tabular}{|c|c|c|c|c|c|}
\hline Case 4 & $\begin{array}{c}\text { Day 1 } \\
\mathbf{1 1 : 5 0}\end{array}$ & $\begin{array}{c}\text { Day 1 } \\
\mathbf{1 3 : 3 2}\end{array}$ & $\begin{array}{c}\text { Day 2 } \\
\mathbf{5 : 0 1}\end{array}$ & $\begin{array}{c}\text { Day 3 } \\
\mathbf{9 : 2 8}\end{array}$ & $\begin{array}{c}\text { Day 4 } \\
\mathbf{3 : 5 0}\end{array}$ \\
\hline Creatinine (mg/dL) & 3.49 & & 2.76 & 2.09 & 1.51 \\
\hline PTH (intact) pg/dL & & 135 & & & 60 \\
\hline Calcium (mg/dL) & 10.7 & & 9.9 & 9.4 & 8.2 \\
\hline Phosphorus (mg/dL) & & & & 1.1 & \\
\hline Potassium (mg/dL) & 4.6 & & 3.8 & 3.8 & 3.5 \\
\hline
\end{tabular}

Table 4: A 57 year-old female with a history of seizures, CVA and hypothyroidism.

\begin{tabular}{|c|c|c|c|c|}
\hline Case 5 & Day 1 & Day 2 & Day 3 & Day 3 \\
& $\mathbf{1 8 : 1 7}$ & $\mathbf{1 4 : 4 1}$ & $\mathbf{4 : 0 5}$ & $\mathbf{1 5 : 1 6}$ \\
\hline Creatinine (mg/dL) & 3.24 & 5.55 & 6.36 & 7.02 \\
\hline PTH(intact) pg/dL & & & 332 & \\
\hline Calcium (mg/dL) & 6.7 & 7.3 & 6.7 & 6.7 \\
\hline Phosphorus (mg/dL) & 5.2 & 5.2 & 6.3 & 2.2 \\
\hline Potassium (mg/dL) & 6 & 5.8 & 4.8 & 4.7 \\
\hline
\end{tabular}

Table 5: A 34 year-old male with a history of alcohol abuse.

\begin{tabular}{|c|c|c|c|c|c|c|c|}
\hline Case 6 & $\begin{array}{c}\text { Day 1 } \\
\mathbf{1 9 : 3 0}\end{array}$ & $\begin{array}{c}\text { Day2 } \\
\mathbf{1 1 : 4 0}\end{array}$ & $\begin{array}{c}\text { Day 2 } \\
\mathbf{1 4 : 5 1}\end{array}$ & $\begin{array}{c}\text { Day 2 } \\
\mathbf{1 7 : 0 0}\end{array}$ & $\begin{array}{c}\text { Day 3 } \\
\mathbf{1 8 : 0 0}\end{array}$ & $\begin{array}{c}\text { Day 4 } \\
\mathbf{5 : 0 0}\end{array}$ & $\begin{array}{c}\text { Day 5 } \\
\mathbf{5 : 2 0}\end{array}$ \\
\hline Creatinine (mg/dL) & 3.4 & 4.4 & 3.89 & 5.05 & & 6.11 & 7.25 \\
\hline BUN (mg/dL) & 28 & 46 & 35 & 47 & & 58 & 65 \\
\hline PTH (intact) pg/dL & & 561 & & & 891 & & 493 \\
\hline Calcium (mg/dL) & 7.2 & 5.9 & 6.8 & 6.5 & & & 7 \\
\hline $\begin{array}{c}\text { Phosphorus (mg/dL) } \\
\text { 25-Hydroxy Vitamin D } \\
\text { (ng/mL) }\end{array}$ & & 4.2 & & & & & \\
\hline
\end{tabular}

Table 6: A 34 year-old white male who was diagnosed with rhabdomyolysis due to drug intoxication.

\section{Case 3}

A 55 year-old female with no history of renal disease presented with a 2-3 day history of right upper quadrant pain and dizziness. She was referred for renal consult after emergency department evaluation revealed hypotension and elevated BUN and creatinine. Further assessment revealed acute kidney injury, most likely secondary to volume depletion. Urine electrolytes were monitored and the patient was administered IV fluids and pressors. Subsequent labs revealed elevated i-PTH in the setting of hypocalcemia. Soon after the hypotension was corrected the patient's creatinine began to decline. The patient eventually regained renal function (Table 3 ).

\section{Case 4}

A 57 year-old female with a history of seizures, CVA and hypothyroidism arrived at the emergency department from a nursing home with complaints of weakness and fever. She was referred for renal consult after urinalysis revealed proteinuria, hematuria, and bacteriuria. Further assessment, suggested acute renal injury. In addition to creatinine, i-PTH was discovered to be elevated. After several days of IV fluids, the patient began to regain renal function. The creatinine began to decline and the PTH followed in a similar manner (Table 4).

\section{Case 5}

A 34 year-old male with a history of alcohol abuse presented after being found unresponsive by his family. He was brought to the emergency department where he was observed to have a high creatinine. Renal consult was obtained and the patient was diagnosed with acute renal failure likely secondary to rhabdomyolysis and volume depletion. Despite supportive treatment, the patient remained oliguric. PTH was still markedly elevated $(332 \mathrm{pg} / \mathrm{ml})$ on day 3 . The patient was placed on dialysis and began to recover over the next several weeks (Table 5).

\section{Case 6 final report}

We describe the case of a 34 year-old white male who presented to the emergency department after being found unresponsive at home. The patient was diagnosed with rhabdomyolysis due to drug intoxication and was admitted to the ICU. The initial serum chemistry values revealed elevated serum potassium at $6.7 \mathrm{mg} / \mathrm{dL}$ and total CK of more than 124,000. BUN and creatinine were $24 \mathrm{mg} / \mathrm{dL}$ and $3.4 \mathrm{~g} /$ day respectively. Calcium was $7.2 \mathrm{mg} / \mathrm{dL}$ and ALT was $80 \mathrm{U} / \mathrm{L}$. Subsequent assessments suggested acute kidney injury (AKI) likely due to rhabdomyolysis. Pre-renal azotemia was suspected due to the high hemoglobin and hematocrit, as well as the concentrated urine.

At this time it was decided that the patient would continue to receive aggressive IV hydration. Renal replacement therapy would not be necessary unless the patient remained oliguric. Serum BUN and creatinine were reviewed continuously and electrolytes were monitored. PTH (intact) and calcium were also assessed.

Despite supportive therapy, PTH remained significantly elevated on day 5. Additionally, the observed PTH elevation was not correlated with a significant increase in serum calcium. The relevant clinical data are given in (Table 6) (Figures 1-4).

\section{Discussion}

Our report of six cases at the local community hospital suggests that a high creatinine observed in CKD is often associated with a persistently elevated PTH. In contrast, patients with a high creatinine as a result of AKI that resolves quickly tend not to have persistently high PTH. Cases 1-4 represent the typical clinical picture of PTH elevation in AKI. In these cases, it is likely that a hypocalcemia-induced increase of plasma parathyroid hormone in early ARF is followed by a downregulation of PTH during the renal function recovery period [19-22]. It seems that the exception to this rule is when the PTH on presentation 


\section{i-PTH, Calcium \& Phosphorus Metabolism}

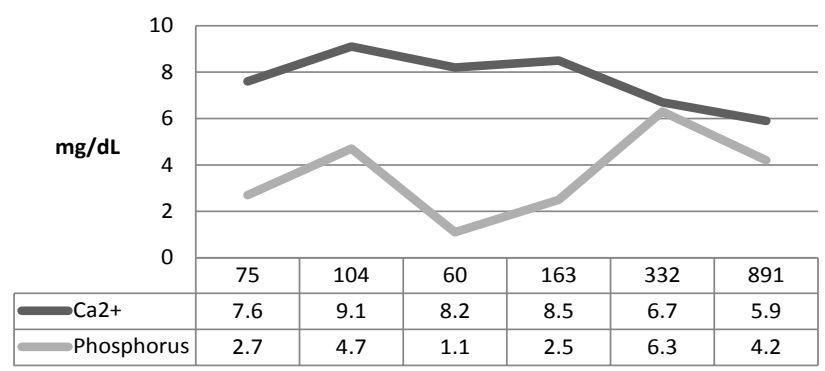

Figure 1: i-PTH, calcium \& phosphorus metabolism.

\section{Creatinine, i-PTH, Calcium \& Phosphorus Metabolism}

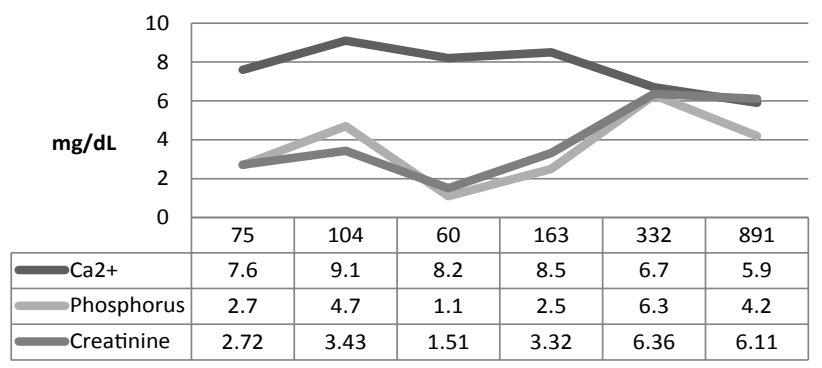

Figure 2: Creatinine, i-PTH, calcium \& phosphorus metabolism.

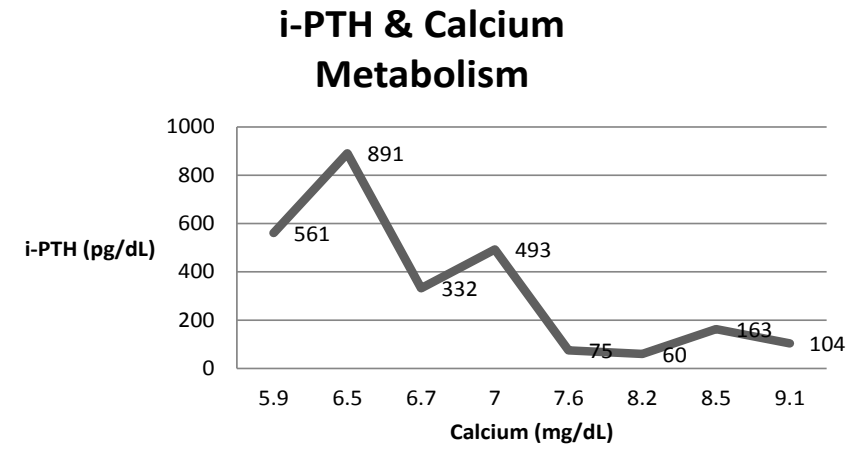

Figure 3: i-PTH \& calcium metabolism.

\section{i-PTH \& Phosphorus}

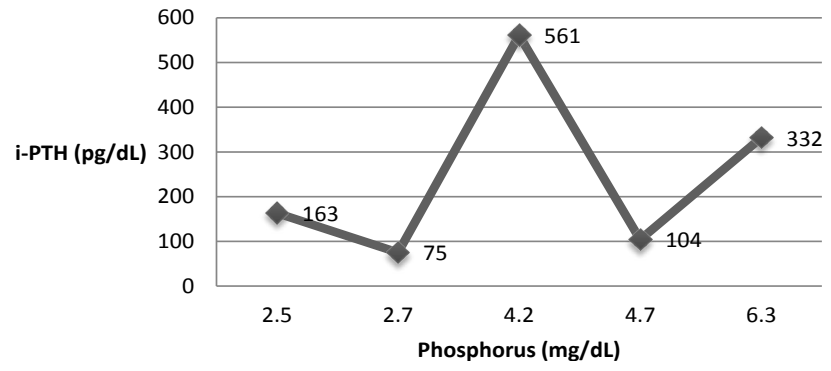

Figure 4: i-PTH \& phosphorus metabolism. is above $250-300 \mathrm{pg} / \mathrm{ml}$ as observed in cases 5 and 6 . In such patients with AKI and severe hyperparathyroidism, PTH may remain elevated despite recovering renal function.

In such rare cases, it seems that the prolonged duration of PTH elevation may be due to rhabdomyolysis and the persistent hypocalcemia observed in these patients. Although cases 5 and 6 provide insight into a unique presentation, further review is required. Specifically, the role of hyperphosphatemia and elevated creatine phosphokinase provoke further questions on the topic.

In similar cases of continued PTH elevation, several different wellstudied markers should be employed to differentiate CKD from AKI, as prompt supportive therapy can be life saving. Alternative markers such as carbamylated hemoglobin may have a diagnostic role in discerning the two conditions $[5,6]$. Notably, it has shown acceptable sensitivity and positive predictive value in dividing patients with potentially reversible renal injury from those with CKD [6]. Additional markers such as serum 1, 5-anhydroglucitol (1,5-AG) may also be useful in such cases as elevated levels correlate well with AKI [23].

Furthermore, we propose that intact PTH should be more routinely screened in patients presenting with acute kidney injury. Despite showing a sensitivity of $88 \%$ and a specificity of $89 \%$ in dividing patients with CKD and AKI [24], a recent study indicated that $\mathrm{PTH}$ was evaluated in only $10 \%$ of patients presenting with presumed AKI [25]. Thus it is likely that increased utilization of this commonly ignored hormone may serve as a simple and inexpensive adjunct in making a more accurate diagnosis.

While CKD has long been associated with SHPT, there is a paucity of literature discussing similar sequela in the setting of AKI. However, the majority of existing literature regarding SHPT in acute kidney injury examines a subset of patients with rhabdomyolysis induced renal failure. In such cases, patients often present with biphasic calcium metabolism consisting of hypocalcemia during the oliguric phase, followed by hypercalcemia in the diuretic phase [26].

Several mechanisms have been proposed to contribute to SHPT in such patients. Of these, skeletal resistance to PTH remains an important common theme [27-29]. Although the precise mechanism for the resistance remains mostly unknown, recent data suggests that elevated FGF-23 may be largely responsible. FGF-23 has been proposed to play a role in both decreasing renal production of calcitriol as well as diminishing skeletal responsiveness to PTH [30]. Additionally calcium sequestration in skeletal muscle may trigger SHPT in these patients [31].

\section{Conclusion}

Continued hyperparathyroidism is a common complication of chronic kidney disease, but is rarely observed in acute kidney injury. Therefore, some degree of suspicion for AKI should be reserved particularly in the patient with elevated PTH despite declining creatinine. Further, we encourage the use of PTH as an inexpensive and readily available marker for differentiating AKI and CKD. Although this case provides an unusual presentation of a common clinical finding, this report is little more than a starting point. Further cases should be reviewed in order to provide a more accurate diagnostic picture and improved treatment plan.

\section{References}

1. Agarwal R, Afzalpurkar R, Fordtran JS (1994) Pathophysiology of potassium absorption and secretion by the human intestine. Gastroenterology 107: 548571. 
Citation: Cavayero C, Kar SM, Kar PM (2014) Blast from the Past-using PTH to Differentiate Acute versus Chronic Kidney Disease. J Nephrol Ther 5: 191. doi:10.4172/2161-0959.1000191

Page 4 of 4

2. Sorensen MV, Matos JE, Praetorius HA, Leipziger J (2010) Colonic potassium handling. Pflugers Arch 459: 645-656.

3. Hayslett JP, Binder HJ (1982) Mechanism of potassium adaptation. Am J Physiol 243: F103-112.

4. Mathialahan T, Maclennan KA, Sandle LN, Verbeke C, Sandle GI (2005) Enhanced large intestinal potassium permeability in end-stage renal disease. J Pathol 206: 46-51.

5. Tasanarong A, Seublinvong T, Eiam-Ong S (2002) The role of carbamylated hemoglobin in identifying acute and chronic renal failure. J Med Assoc Tha 85: $462-469$

6. Davenport A, Jones SR, Goel S, Astley JP, Hartog M (1993) Differentiation of acute from chronic renal impairment by detection of carbamylated haemoglobin. Lancet 341: 1614-1617.

7. Lehmann G, Stein G, Hüller M, Schemer R, Ramakrishnan K, et al. (2005) Specific measurement of PTH (1-84) in various forms of renal osteodystrophy (ROD) as assessed by bone histomorphometry. Kidney Int 68: 1206-1214.

8. Tomasello $S$ (2008) Secondary hyperparathyroidism and chronic kidney disease. Diabetes Spectrum 21: 19-25.

9. Cunningham J, Locatelli F, Rodriguez M (2011) Secondary hyperparathyroidism: pathogenesis, disease progression, and therapeutic options. Clinical Journal of the American Society of Nephrology 6: 913-921.

10. Rodríguez M, López I, Muñoz J, Aguilera-Tejero E, Almaden Y (2012) FGF23 and mineral metabolism, implications in CKD-MBD. Nefrologia 32: 275-278.

11. Hudson JQ (2006) Secondary hyperparathyroidism in chronic kidney disease: focus on clinical consequences and vitamin D therapies. Ann Pharmacother 40: 1584-1593

12. Christov M, Waikar SS, Pereira RC, Havasi A, Leaf DE, et al. (2013) Plasma FGF23 levels increase rapidly after acute kidney injury. Kidney Int 84: 776-785.

13. Zhang M, Hsu R, Hsu CY, Kordesch K, Nicasio E, et al. (2011) FGF-23 and PTH levels in patients with acute kidney injury: A cross-sectional case series study. Ann Intensive Care 1: 21

14. Cozzolino M, Malindretos $P(2010)$ The role of vitamin $D$ receptor activation in chronic kidney disease. Hippokratia 14: 7-9.

15. Geara AS, Castellanos MR, Bassil C, Schuller-Levis G, Park E, et al. (2010) Effects of parathyroid hormone on immune function. Clin Dev Immunol 2010.

16. van Ballegooijen AJ, Reinders I, Visser M, Dekker JM, Nijpels G, et al. (2013) Serum parathyroid hormone in relation to all-cause and cardiovascular mortality: the Hoorn study. J Clin Endocrinol Metab 98: E638-645.

17. Cooper JD, Lazarowitz VC, Arieff Al (1978) Neurodiagnostic abnormalities in patients with acute renal failure. J Clin Invest 61: 1448-1455.

18. Cheng SP, Liu CL, Liu TP, Hsu YC, Lee JJ (2014) Association between parathyroid hormone levels and inflammatory markers among US adults. Mediators Inflamm 2014: 709024.

19. Meneghini LF, Oster JR, Camacho JR, Gkonos PJ, Roos BA (1993) Hypercalcemia in association with acute renal failure and rhabdomyolysis. Case report and literature review. Miner Electrolyte Metab 19: 1-16.

20. Kokot F, Wiecek A, Grzeszczak W (1993) Plasma parathyroid hormone and erythropoietin levels in patients with noninflammatory acute renal failure. Int Urol Nephrol 25: 89-96.
21. Fuss M, Bagon J, Dupont E, Manderlier T, Brauman H, et al. (1978) Parathyroid hormone and calcium blood levels in acute renal failure. With special reference to one patient developing transient hypercalcemia. Nephron 20: 196-202.

22. Graziani G, Calvetta A, Cucchiari D, Valaperta S, Montanelli A (2011) Lifethreatening hypercalcemia in patients with rhabdomyolysis-induced oliguric acute renal failure. J Nephrol 24: 128-131.

23. Yamada H, Hishida A, Kato A, Yoneyama T (1996) 1,5-anhydroglucitol as a marker for the differential diagnosis of acute and chronic renal failure. Nephron 73: 707-709.

24. Ozmen S, Danis R, Akin D, Cil T, Yazanel O (2007) Parathyroid hormone as a marker for the differential diagnosis of acute and chronic renal failure. Ren Fail 29: 509-512.

25. Matheny ME, Peterson JF, Eden SK, Hung AM, Speroff T, et al. (2014) Laboratory test surveillance following acute kidney injury. PLoS One 9: e103746.

26. Cetinkaya R, Uyanik A, Keles M, Bilen Y (2009) The rhabdomyolysis related acute renal failure and biphasic calcium metabolism. Pak J Med Sci 25: 152154

27. Massry SG, Stein R, Garty J, Arieff Al, Coburn JW, et al. (1976) Skeletal resistance to the calcemic action of parathyroid hormone in uremia: role of 1,25 $(\mathrm{OH}) 2$ D3. Kidney Int 9: 467-474.

28. Llach F, Massry SG, Singer FR, Kurokawa K, Kaye JH, et al. (1975) Skeletal resistance to endogenous parathyroid hormone in patients with early renal failure. A possible cause for secondary hyperparathyroidism. J Clin Endocrinol Metab 41: 339-345.

29. Somerville PJ, Kaye M (1978) Resistance to parathyroid hormone in renal failure: role of vitamin D metabolites. Kidney Int 14: $245-254$

30. Gutierrez O, Isakova T, Rhee E, Shah A, Holmes J, et al. (2005) Fibroblas growth factor-23 mitigates hyperphosphatemia but accentuates calcitriol deficiency in chronic kidney disease. J Am Soc Nephrol 16: 2205-2215.

31. Leaf DE, Wolf M, Stern L (2010) Elevated FGF-23 in a patient with rhabdomyolysis-induced acute kidney injury. Nephrol Dial Transplant 25: 13351337. 